\title{
TCAM Razor: A Systematic Approach Towards Minimizing Packet Classifiers in TCAMs
}

\author{
Chad R. Meiners Alex X. Liu Eric Torng Yun Zhou \\ Department of Computer Science and Engineering \\ Michigan State University \\ East Lansing, MI 48823, U.S.A. \\ \{meinersc, alexliu, torng, zhouyun\}@cse.msu.edu
}

\begin{abstract}
Packet classification is the core mechanism that enables many networking services on the Internet such as firewall packet filtering and traffic accounting. Using Ternary Content Addressable Memories (TCAMs) to perform high-speed packet classification has become the de facto standard in industry. TCAMs classify packets in constant time by comparing a packet with all classification rules of ternary encoding in parallel.

Despite their high speed, TCAMs suffer from the well-known prefix expansion problem. As packet classification rules usually have fields specified as intervals, converting such rules to TCAMcompatible rules may result in an explosive increase in the number of rules. This is not a problem if TCAMs have large capacities. Unfortunately, TCAMs have very limited capacity, and more rules means more power consumption and more heat generation for TCAMs. Even worse, the number of rules in packet classifiers have been increasing rapidly with the growing number of services deployed on the internet.

To address the prefix expansion problem of TCAMs, we consider the following problem: given a packet classifier, how can we generate another semantically equivalent packet classifier that requires the least number of TCAM entries? In this paper, we propose a systematic approach, the TCAM Razor, that is effective, efficient, and practical. In terms of effectiveness, our TCAM Razor prototype achieves a total compression ratio of $3.9 \%$, which is significantly better than the previously published best result of $54 \%$. In terms of efficiency, our TCAM Razor prototype runs in seconds, even for large packet classifiers. Finally, in terms of practicality, our TCAM Razor approach can be easily deployed as it does not require any modification to existing packet classification systems, unlike many previous prefix expansion solutions.
\end{abstract}

\section{INTRODUCTION}

Packet classification, which has been widely deployed on the Internet, is the core mechanism that enables routers to perform many networking services such as firewall packet filtering, virtual private networks (VPNs), network address translation (NAT), quality of service (QoS), load balancing, traffic accounting and monitoring, differentiated services (Diffserv), etc. As more services are deployed on the Internet, packet classification grows in demand and importance.

The function of a packet classification system is to map each packet to a decision (i.e., action) according to a sequence (i.e., ordered list) of rules, which is called a packet classifier. Each rule in a packet classifier has a predicate over some packet header fields and a decision to be performed upon the packets that match the predicate. To resolve possible conflicts among rules in a classifier, the decision for each packet is the decision of the first (i.e., highest priority) rule that the packet matches. Table I shows an example packet classifier of two rules. The format of these rules is based upon the format used in Access Control Lists on Cisco routers.

\section{A. Motivation}

There are two types of packet classification schemes: softwarebased and hardware-based. Many software-based packet classification algorithms and techniques have been proposed in the past decade (e.g., [6], [7], [10], [12], [15], [21], [22], [24], [28], [29]). Based on complexity bounds from computational geometry [20], for packet classification with $n$ rules and $d>3$ fields, the "best" software-based packet classification algorithms use either $O\left(n^{d}\right)$ space and $O(\log n)$ time or $O(n)$ space and $O\left(\log ^{d-1} n\right)$ time. Many software-based solutions are either too slow (such as linear search) or too memory intensive (such as RFC [12]). Decisiontree based packet classifiers, which were pioneered by Woo [29] and Gupta and McKeown [13], seem to achieve better time-space tradeoffs. However, they may not work as well in the future as they have exploited statistical characteristics of packets classifiers to achieve the above time-space tradeoffs, and it has been observed that these statistical characteristics are changing [16].

Due to the inherent limitations of software-based packet classification algorithms, more and more packet classification systems are hardware-based; specifically, most packet classification systems now use Ternary Content Addressable Memories (TCAMs). A TCAM is a memory chip where each entry can store a packet classification rule that is encoded in ternary format. Given a packet, the TCAM hardware can compare the packet with all stored rules in parallel and then return the decision of the first rule that the packet matches. Thus, it takes $O(1)$ time to find the decision for any given packet. Because of their high speed, TCAMs have become the de facto industrial standard for high speed packet classification [1]-[3], [16]. In 2003, most packet classification devices shipped were TCAM-based [4]. More than 6 million TCAM devices were deployed worldwide in 2004 [4].

Despite their high speed, TCAMs have their own limitations with respect to packet classification.

a) Prefix expansion: TCAMs can only store rules that are encoded in ternary format. In a typical packet classification rule, source IP address, destination IP address, and protocol type are specified in prefix format, which can be directly stored in TCAMs, but source and destination port numbers are specified in intervals (i.e., ranges), which need to be converted to one or more prefixes before being stored in TCAMs. This can lead to a significant increase in the number of TCAM entries needed to encode a rule. For example, 30 prefixes are needed to represent the single interval $[1,65534]$, so $30 \times 30=900$ TCAM entries are required to represent the single rule $r_{1}$ in Table I.

b) Low capacity: TCAMs have limited capacity. The largest TCAM chip available on the market has $18 \mathrm{Mb}$ while $2 \mathrm{Mb}$ and $1 \mathrm{Mb}$ chips are most popular [4]. Given that each TCAM entry 


\begin{tabular}{|c|ccccc|c|}
\hline Rule & Source IP & Destination IP & Source Port & Destination Port & Protocol & Action \\
\hline$r_{1}$ & $1.2 .3 .0 / 24$ & 192.168 .0 .1 & {$[1,65534]$} & {$[1,65534]$} & TCP & accept \\
$r_{2}$ & $*$ & $*$ & $*$ & $*$ & $*$ & discard \\
\hline
\end{tabular}

TABLE I

AN EXAMPLE PACKET CLASSIFIER

\begin{tabular}{|c|ccccc|c|}
\hline Rule & Source IP & Destination IP & Source Port & Destination Port & Protocol & Action \\
\hline$r_{1}$ & $1.2 .3 .0 / 24$ & 192.168 .0 .1 & 0 & $*$ & $*$ & discard \\
$r_{2}$ & $1.2 .3 .0 / 24$ & 192.168 .0 .1 & 65535 & $*$ & $*$ & discard \\
$r_{3}$ & $1.2 .3 .0 / 24$ & 192.168 .0 .1 & $*$ & 0 & $*$ & discard \\
$r_{4}$ & $1.2 .3 .0 / 24$ & 192.168 .0 .1 & $*$ & 65535 & $*$ & discard \\
$r_{5}$ & $1.2 .3 .0 / 24$ & 192.168 .0 .1 & {$[0,65535]$} & {$[0,65535]$} & TCP & accept \\
$r_{6}$ & $*$ & $*$ & $*$ & $*$ & $*$ & discard \\
\hline
\end{tabular}

TABLE II

TCAM RAZOR OUTPUT FOR THE EXAMPLE PACKET CLASSIFIER IN FIGURE I

has 144 bits and a packet classification rule may have a worst expansion factor of 900 , it is possible that an $18 \mathrm{Mb}$ TCAM chip cannot store all the required entries for a modest packet classifier of only 139 rules. While the worst case may not happen in reality, this is certainly an alarming issue. Furthermore, TCAM capacity is not expected to increase dramatically in the near future due to other limitations that we will discuss next.

c) High power consumption and heat generation: TCAM chips consume large amounts of power and generate large amounts of heat. For example, a 1Mb TCAM chip consumes 15-30 watts of power. Power consumption together with the consequent heat generation is a serious problem for core routers and other networking devices.

d) Large board space occupation: TCAMs occupy much more board space than SRAMs. For networking devices such as routers, area efficiency of the circuit board is a critical issue.

e) High hardware cost: TCAMs are expensive. For example, a $1 \mathrm{Mb}$ TCAM chip costs about $200 \sim 250$ U.S. dollars. TCAM cost is a significant fraction of router cost.

\section{B. The Problem}

In this paper, we consider the following TCAM Minimization Problem: given a packet classifier, how can we generate another semantically equivalent packet classifier that requires the least number of TCAM entries? Two packet classifiers are (semantically) equivalent if and only if they have the same decision for every packet. For example, the two packets classifiers in Tables I and II are equivalent; however, the one in Table I requires 900 TCAM entries, and the one in Table II requires only 6 TCAM entries.

Solving this problem helps to address the limitations of TCAMs. As we reduce the number of TCAM entries required, we can use smaller TCAMs, which results in less board space and less hardware cost. Furthermore, reducing the number of rules in a TCAM directly reduces power consumption and heat generation as the energy consumed by a TCAM grows linearly with the number of ternary rules it stores [30].

\section{Our Solution: TCAM Razor}

While the optimal solution to the above problem is conceivably NP-hard, in this paper, we propose a practical algorithmic solution using three techniques: decision diagrams, dynamic programming, and redundancy removal. Our solution consists of the following four basic steps. First, convert a given packet classifier to a reduced decision diagram, which is the canonical representation of the semantics of the given packet classifier. Second, for every nonterminal node in the decision diagram, minimize the number of prefixes associated with its outgoing edges using dynamic programming. Third, generate rules from the decision diagram. Last, remove redundant rules. As an example, running our algorithms on the packet classifier in Table I will yield the one in Table II.

Our solution is effective, efficient, and practical. In terms of effectiveness, our approach achieves a total compression ratio of $3.9 \%$ on real-life packet classifiers, which is significantly better than the previously published best result of $54 \%$ [8]. In terms of efficiency, our approach runs in seconds, even for large packet classifiers. Finally, in terms of practicality, our approach can be easily deployed as it does not require any modification of existing packet classification systems. In comparison, a number of previous solutions require hardware and architecture modifications to existing packet classification systems, making their adoption by networking manufacturers and ISPs much less likely.

We name our solution "TCAM Razor" following the principle of Occam's razor: "Of two equivalent theories or explanations, all other things being equal, the simpler one is to be preferred." In our context, of all packet classifiers that are equivalent, the one with the least number of TCAM entries is preferred.

The rest of this paper proceeds as follows. We start by reviewing related work in Section II. In Section III, we formally define the TCAM minimization problem and related terms. In Section IV, we discuss the weighted one-dimensional TCAM minimization problem. In Section V, we give a solution to the multi-dimensional TCAM minimization problem, whose optimization techniques are presented in Section VI. In Section VII, we show the experimental results on both real-life and synthetic packet classifiers. Finally, we give concluding remarks in Section VIII.

\section{RELATED WORK}

Many software solutions have been proposed for finding the decision of the first rule that a packet matches in a given packet classifier (e.g., [6], [7], [10], [12], [15], [21], [22], [24], [28], [29]). A comprehensive survey of this work is given in [26].

Recently, hardware packet classification systems based on TCAMs have been widely deployed due to their $O(1)$ classification 
time. This has led to a significant amount of work that explores ways to cope with the well-known prefix expansion problem. These solutions fall into three broad categories: (1) TCAM modification, which requires changing TCAM hardware circuits, (2) range encoding, which does not require changing TCAM hardware circuits, but does require preprocessing for every packet, and (3) classifier minimization, which does not require changing TCAM hardware circuits nor preprocessing for any packet.

TCAM Modification: The basic idea is to modify TCAM circuits for packet classification purposes. For example, Spitznagel et al. proposed adding comparators at each entry level to better accommodate range matching [23]. This is an important research direction. However, any solutions from this research line will not be deployed for many years due to issues of cost and development [16]. Furthermore, changing the ternary nature of TCAMs makes such TCAMs less generally applicable to applications other than packet classification.

Range Encoding: The basic idea is to re-encode intervals that appear in a packet classifier and then store the re-encoded rules in the TCAM. When a packet comes, the packet needs to be preprocessed according to the re-encoding scheme such that the packet, after preprocessing, can be used as a search key for the TCAM. Several range encoding schemes have been proposed [16], [19], [27]. While the TCAM circuit does not need to be modified to implement range encoding, the system hardware does need to be reconfigured to allow for preprocessing of packets, and the delay caused by packet preprocessing could be problematic.

Classifier Minimization: The basic idea is to convert a given packet classifier to another semantically equivalent packet classifier that requires fewer TCAM entries. These solutions are the most likely to be deployed by networking vendors and ISPs because they require no changes to TCAM hardware or existing packet classification systems and incur no preprocessing overhead for packets. Our work, along with [5], [8], [9], [18], [25], falls into this category.

Three papers focus on one-dimensional and two dimensional packet classifiers. Draves et al. proposed an optimal solution for one-dimensional packet classifiers in the context of minimizing routing tables in [9]. Subsequently, in the same context of minimizing routing tables, Suri et al. proposed an optimal dynamic programming solution for one-dimensional packet classifiers. They also observed that a generalization of the dynamic program was optimal for two-dimensional packet classifiers in which two rules either are non-overlapping or one contains the other geometrically [25]. Suri et al. noted that their dynamic program would not be optimal for packet classifiers with more than 2 dimensions. In our studies, we have extended and implemented Suri et al.'s algorithm to minimize 5-dimensional packet classifiers. Unfortunately, the extended algorithm is prohibitively slow even for a packet classifier with just a few rules. Recently, Applegate et al. proposed an optimal solution for packet classifiers with two dimensions in which each rule must have one field specified as the whole domain of the field and there are only 2 decisions [5].

Only two papers have considered minimizing packet classifiers with more than 2 dimensions. In [18], Liu and Gouda proposed the first algorithm to remove all the redundant rules in a packet classifier, which consequently reduces the number of TCAM entries needed. In [8], Dong et al. observed that both expanding and trimming intervals so that they correspond to prefixes can result in fewer TCAM entries. Our TCAM Razor handles these special cases and more. As we demonstrate in Section VII, TCAM Razor significantly outperforms Liu and Gouda's redundancy removal technique and Dong et al.'s heuristics. For example, the total compression ratios for TCAM Razor, redundancy removal, and Dong et al.'s scheme are $3.9 \%, 35 \%$, and $54 \%$ respectively. Furthermore, the running time of Dong et al.'s techniques are not reported. In comparison, TCAM Razor runs in seconds on a mediocre desktop $\mathrm{PC}$, even for large packet classifiers.

It is not surprising that TCAM Razor outperforms the heuristics of Dong et al.. First, although TCAM Razor and Dong et al.'s heuristics both process packet classifiers one dimension at a time, TCAM Razor is guaranteed to achieve optimal compression on that dimension, but Dong et al.'s heuristics are not. Specifically, TCAM Razor handles all the special cases that Dong et al. identify in a systematic fashion. Second, while packet classifier semantics are highly dependent on rule order given their first-match semantics, TCAM Razor reduces the influence of rule order by converting the given packet classifier to a reduced decision diagram, which is a canonical representation of the given packet classifier. On the other hand, Dong et al. process rules in their original order, looking at one rule at a time for optimization possibilities.

\section{FORMAL DEFINITIONS}

We now formally define the concepts of fields, packets, packet classifiers, and the TCAM Minimization Problem. A field $F_{i}$ is a variable of finite length (i.e., of a finite number of bits). The domain of field $F_{i}$ of $w$ bits, denoted $D\left(F_{i}\right)$, is $\left[0,2^{w}-1\right]$. A packet over the $d$ fields $F_{1}, \cdots, F_{d}$ is a $d$-tuple $\left(p_{1}, \cdots, p_{d}\right)$ where each $p_{i}$ $(1 \leq i \leq d)$ is an element of $D\left(F_{i}\right)$. Packet classifiers usually check the following five fields: source IP address, destination IP address, source port number, destination port number, and protocol type. The length of these packet fields are $32,32,16,16$, and 8 respectively. We use $\Sigma$ to denote the set of all packets over fields $F_{1}, \cdots, F_{d}$. It follows that $\Sigma$ is a finite set and $|\Sigma|=\left|D\left(F_{1}\right)\right| \times$ $\cdots \times\left|D\left(F_{d}\right)\right|$, where $|\Sigma|$ denotes the number of elements in set $\Sigma$ and $\left|D\left(F_{i}\right)\right|$ denotes the number of elements in set $D\left(F_{i}\right)$.

A rule has the form $\langle$ predicate $\rangle \rightarrow\langle$ decision $\rangle$. A $\langle$ predicate $\rangle$ defines a set of packets over the fields $F_{1}$ through $F_{d}$, and is specified as $F_{1} \in S_{1} \wedge \cdots \wedge F_{d} \in S_{d}$ where each $S_{i}$ is a subset of $D\left(F_{i}\right)$ and is specified as either a prefix or a nonempty nonnegative integer interval. A prefix $\{0,1\}^{k}\{*\}^{w-k}$ with $k$ leading 0 s or 1 s for a packet field of length $w$ denotes the integer interval $\left[\{0,1\}^{k}\{0\}^{w-k},\{0,1\}^{k}\{1\}^{w-k}\right]$. For example, prefix $01^{* *}$ denotes the interval [0100,0111]. A rule $F_{1} \in S_{1} \wedge \cdots \wedge F_{d} \in S_{d} \rightarrow$ $\langle$ decision $\rangle$ is a prefix rule if and only if each $S_{i}$ is represented as a prefix.

When using a TCAM to implement a packet classifier, we typically require that all rules be prefix rules. However, in a typical packet classifier rule, some fields such as source and destination port numbers are represented as integer intervals rather than a prefix. This leads to prefix expansion, the process of converting a rule that may have fields represented as integer intervals into a prefix rule. In prefix expansion, each field of a rule is first expanded separately. The goal is to find a minimum set of prefixes such that the union of the prefixes corresponds to the integer interval. For example, if one 3-bit field of a rule is the integer interval $[1,7]$, a corresponding minimum set of prefixes would be $001,01 *, 10 *$, 110. The worst-case prefix expansion of a $w$-bit integer interval results in a set containing $2 w-2$ prefixes [14]. The next step is to compute the cross product of each set of prefixes for each field, resulting in a potentially large number of prefix rules. In Section I, the prefix expansion of rule $r_{1}$ in Table I resulted in $30 \times 30=900$ prefix rules. 
A packet $\left(p_{1}, \cdots, p_{d}\right)$ matches a predicate $F_{1} \in S_{1} \wedge \cdots \wedge F_{d} \in$ $S_{d}$ and the corresponding rule if and only if the condition $p_{1} \in$ $S_{1} \wedge \cdots \wedge p_{d} \in S_{d}$ holds. We use $\alpha$ to denote the set of possible values that $\langle$ decision $\rangle$ can be. Typical elements of $\alpha$ include accept, discard, accept with logging, and discard with logging.

A sequence of rules $\left\langle r_{1}, \cdots, r_{n}\right\rangle$ is complete if and only if for any packet $p$, there is at least one rule in the sequence that $p$ matches. To ensure that a sequence of rules is complete and thus a packet classifier, the predicate of the last rule is usually specified as $F_{1} \in D\left(F_{1}\right) \wedge \cdots F_{d} \in \wedge D\left(F_{d}\right)$. A packet classifier $f$ is a sequence of rules that is complete. The size of $f$, denoted $|f|$, is the number of rules in $f$. A packet classifier $f$ is a prefix packet classifier if and only if every rule in $f$ is a prefix rule.

Two rules in a packet classifier may overlap; that is, there exists at least one packet that matches both rules. Furthermore, two rules in a packet classifier may conflict; that is, the two rules not only overlap but also have different decisions. Packet classifiers typically resolve conflicts by employing a first-match resolution strategy where the decision for a packet $p$ is the decision of the first (i.e., highest priority) rule that $p$ matches in $f$. The decision that packet classifier $f$ makes for packet $p$ is denoted $f(p)$.

We can think of a packet classifier $f$ as defining a many-to-one mapping function from $\Sigma$ to $\alpha$, where $\Sigma$ denotes the set of all possible packets and $\alpha$ denotes the set of all possible decisions. Two packet classifiers $f_{1}$ and $f_{2}$ are equivalent, denoted $f_{1} \equiv f_{2}$, if and only if they define the same mapping function from $\Sigma$ to $\alpha$; that is, for any packet $p \in \Sigma$, we have $f_{1}(p)=f_{2}(p)$. For any packet classifier $f$, we use $\{f\}$ to denote the set of packet classifiers that are equivalent to $f$. Now we are ready to define the TCAM Minimization Problem.

Definition 3.1 (TCAM Minimization Problem): Given a packet classifier $f_{1}$, find a prefix packet classifier $f_{2} \in\left\{f_{1}\right\}$ such that for any prefix packet classifier $f \in\left\{f_{1}\right\}$, the condition $\left|f_{2}\right| \leq|f|$ holds.

\section{ONE-DIMENSIONAL TCAM MINIMIZATION}

We first consider the special problem of weighted one-dimensional TCAM minimization, whose solution is used in the next section as a building block for multidimensional TCAM minimization. Given a one-dimensional packet classifier $f$ of $n$ prefix rules $\left\langle r_{1}, r_{2}, \cdots, r_{n}\right\rangle$, where $\left\{\operatorname{Decision}\left(r_{1}\right), \operatorname{Decision}\left(r_{2}\right), \cdots, \operatorname{Decision}\left(r_{n}\right)\right\}=$ $\left\{d_{1}, d_{2}, \cdots, d_{z}\right\}$ and each decision $d_{i}$ is associated with a cost $\operatorname{Cost}\left(d_{i}\right)$ (for $1 \leq i \leq z$ ), we define the cost of packet classifier $f$ as follows:

$$
\operatorname{Cost}(f)=\sum_{i=1}^{n} \operatorname{Cost}\left(\operatorname{Decision}\left(r_{i}\right)\right)
$$

Based upon the above definition, the problem of weighted onedimensional TCAM minimization is stated as follows.

Definition 4.1: (Weighted One-dimensional TCAM Minimization Problem) Given a one-dimensional packet classifier $f_{1}$ where each decision is associated with a cost, find a prefix packet classifier $f_{2} \in\left\{f_{1}\right\}$ such that for any prefix packet classifier $f \in\left\{f_{1}\right\}$, the condition $\operatorname{Cost}\left(f_{2}\right) \leq \operatorname{Cost}(f)$ holds.

The problem of one-dimensional TCAM minimization (with uniform cost) has been studied in [9], [25] in the context of compressing routing tables. In this paper, we extend the dynamic programming solution in [25] to solve the weighted one-dimensional TCAM minimization. There are three key observations:

1) For any one-dimensional packet classifier $f$ on $\{*\}^{w}$, we can always change the predicate of the last rule to be $\{*\}^{w}$ without changing the semantics of the packet classifier. This follows from the completeness property of packet classifiers.

2) Consider any one-dimensional packet classifier $f$ on $\{*\}^{w}$. Let $f^{\prime}$ be $f$ appended with rule $\{*\}^{w} \rightarrow d$, where $d$ can be any decision. The observation is that $f \equiv f^{\prime}$. This is because the new rule is redundant in $f^{\prime}$ since $f$ must be complete. A rule in a packet classifier is redundant if and only if removing the rule from the packet classifier does not change the semantics of the packet classifier.

3) Any prefix $\{0,1\}^{k}\{*\}^{w-k}(1 \leq k \leq w)$ satisfies one of the following three mutually exclusive conditions:

a) $\{0,1\}^{k}\{*\}^{w-k} \subseteq 0\{*\}^{w-1}$,

b) $\{0,1\}^{k}\{*\}^{w-k} \subseteq 1\{*\}^{w-1}$,

c) $\{0,1\}^{k}\{*\}^{w-k}=\{*\}^{w}$.

This property allows us to divide a problem on $\{0,1\}^{k}\{*\}^{w-k}$ into two sub-problems: one on $\{0,1\}^{k} 0\{*\}^{w-k-1}$, and the other one on $\{0,1\}^{k} 1\{*\}^{w-k-1}$.

This divide-and-conquer strategy can be applied recursively.

Based on the above three observations, we formulate an optimal dynamic programming solution to the weighted one-dimensional TCAM minimization problem.

Let $\mathcal{P}$ denote a prefix $\{0,1\}^{k}\{*\}^{w-k}$. We use $\underline{\mathcal{P}}$ to denote the prefix $\{0,1\}^{k} 0\{*\}^{w-k-1}$, and $\overline{\mathcal{P}}$ to denote the prefix $\{0,1\}^{k} 1\{*\}^{w-k-1}$.

Given a one-dimensional packet classifier $f$ on $\{*\}^{w}$, we use $f_{\mathcal{P}}$ to denote a packet classifier on $\mathcal{P}$ such that for any $x \in \mathcal{P}$, $f_{\mathcal{P}}(x)=f(x)$, and we use $f_{\mathcal{P}}^{d}$ to denote a similar packet classifier on $\mathcal{P}$ with the additional restriction that the final decision is $d$.

We use $C\left(f_{\mathcal{P}}\right)$ to denote the minimum cost of a packet classifier $t$ that is equivalent to $f_{\mathcal{P}}$, and we use $C\left(f_{\mathcal{P}}^{d}\right)$ to denote the minimum cost of a packet classifier $t^{\prime}$ that is equivalent to $f_{\mathcal{P}}$ and the decision of the last rule in $t^{\prime}$ is $d$.

Given a one-dimensional packet classifier $f$ on $\{*\}^{w}$ and a prefix $\mathcal{P}$ where $\mathcal{P} \subseteq\{*\}^{w}, f$ is consistent on $\mathcal{P}$ if and only if $\forall x, y \in \mathcal{P}$, $f(x)=f(y)$.

Our dynamic programming solution to the weighted onedimensional TCAM minimization problem is based on the following theorem. The proof of the theorem shows how to divide a problem into sub-problems and how to combine solutions to subproblems into a solution to the original problem.

Theorem 4.1: Given a one-dimensional packet classifier $f$ on $\{*\}^{w}$, a prefix $\mathcal{P}$ where $\mathcal{P} \subseteq\{*\}^{w}$, the set of all possible decisions $\left\{d_{1}, d_{2}, \cdots, d_{z}\right\}$ where each decision $d_{i}$ has a cost $w_{d_{i}}(1 \leq i \leq z)$, we have that

$$
C\left(f_{\mathcal{P}}\right)=\min _{i=1}^{z} C\left(f_{\mathcal{P}}^{d_{i}}\right)
$$

where each $C\left(f_{\mathcal{P}}^{d_{i}}\right)$ is calculated as follows: (1) If $f$ is consistent on $\mathcal{P}$, then

$$
C\left(f_{\mathcal{P}}^{d_{i}}\right)= \begin{cases}w_{f(x)} & \text { if } f(x)=d_{i} \\ w_{f(x)}+w_{d_{i}} & \text { if } f(x) \neq d_{i}\end{cases}
$$

(2) If $f$ is not consistent on $\mathcal{P}$, then

$$
C\left(f_{\mathcal{P}}^{d_{i}}\right)=\min \left\{\begin{array}{l}
C\left(f_{\mathcal{P}}^{d_{1}}\right)+C\left(f_{\overline{\mathcal{P}}}^{d_{1}}\right)-w_{d_{1}}+w_{d_{i}}, \\
\ldots\left(f_{\mathcal{P}}^{d_{i-1}}\right)+C\left(f_{\overline{\mathcal{P}}}^{d_{i-1}}\right)-w_{d_{i-1}}+w_{d_{i}}, \\
C\left(f_{\mathcal{P}}^{d_{i}}\right)+C\left(f_{\overline{\mathcal{P}}}^{d_{i}}\right)-w_{d_{i}}, \\
C\left(f_{\mathcal{P}}^{d_{i+1}}\right)+C\left(f_{\overline{\mathcal{P}}}^{d_{i+1}}\right)-w_{d_{i+1}}+w_{d_{i}}, \\
\ldots\left(f_{\underline{\mathcal{P}}}^{d_{z}}\right)+C\left(f_{\overline{\mathcal{P}}}^{d_{z}}\right)-w_{d_{a}}+w_{d_{i}}
\end{array}\right.
$$

Proof: (1) The base case of the recursion is when $f$ is consistent on $\mathcal{P}$. In this case, the minimum cost prefix packet classifier in $\left\{f_{\mathcal{P}}\right\}$ is clearly $\langle\mathcal{P} \rightarrow f(x)\rangle$, and the cost of this packet classifier is $w_{f(x)}$. Furthermore, for $d_{i} \neq f(x)$, the minimum cost 
prefix packet classifier in $\left\{f_{\mathcal{P}}\right\}$ with decision $d_{i}$ in the last rule is $\left\langle\mathcal{P} \rightarrow f(x), \mathcal{P} \rightarrow d_{i}\right\rangle$ where the second rule is redundant. The cost of this packet classifier is $w_{f(x)}+w_{d_{i}}$.

(2) If $f$ is not consistent on $\mathcal{P}$, we divide $\mathcal{P}$ into $\underline{\mathcal{P}}$ and $\overline{\mathcal{P}}$. The crucial observation is that an optimal solution $f^{*}$ to $\left\{f_{\mathcal{P}}\right\}$ is essentially an optimal solution $f_{1}$ to the sub-problem of minimizing $f_{\mathcal{P}}$ appended with an optimal solution $f_{2}$ to the sub-problem of minimizing $f_{\overline{\mathcal{P}}}$. The only interaction that can occur between $f_{1}$ and $f_{2}$ is if their final rules have the same decision, in which case both final rules can be replaced with one final rule covering all of $\mathcal{P}$ with the same decision. Let $d_{x}$ be the decision of the last rule in $f_{1}$ and $d_{y}$ be the decision of the last rule in $f_{2}$. Then we can compose $f^{*}$ whose last rule has decision $d_{i}$ from $f_{1}$ and $f_{2}$ based on the following cases:

(A) $d_{x}=d_{y}=d_{i}$ : In this case, $f$ can be constructed by listing all the rules in $f_{1}$ except the last rule, followed by all the rules in $f_{2}$ except the last rule, and then the last rule $\mathcal{P} \rightarrow d_{i}$. Thus, $\operatorname{Cost}(f)=\operatorname{Cost}\left(f_{1}\right)+\operatorname{Cost}\left(f_{2}\right)-w_{d_{i}}$.

(B) $d_{x}=d_{y} \neq d_{i}$ : In this case, $f$ can be constructed by listing all the rules in $f_{1}$ except the last rule, followed by all the rules in $f_{2}$ except the last rule, then rule $\mathcal{P} \rightarrow d_{x}$, and finally rule $\mathcal{P} \rightarrow d_{i}$ Thus, $\operatorname{Cost}(f)=\operatorname{Cost}\left(f_{1}\right)+\operatorname{Cost}\left(f_{2}\right)-w_{d_{x}}+w_{d_{i}}$.

(C) $d_{x} \neq d_{y}, d_{x}=d_{i}, d_{y} \neq d_{i}$ : We do not need to consider this case because $C\left(f_{\underline{\mathcal{P}}}^{d_{i}}\right)+C\left(f_{\overline{\mathcal{P}}}^{d_{y}}\right)=C\left(f_{\underline{\mathcal{P}}}^{d_{i}}\right)+\left(C\left(f_{\overline{\mathcal{P}}}^{d_{y}}\right)+w_{d_{i}}\right)-w_{d_{i}} \geq$ $C\left(f_{\mathcal{P}}^{d_{i}}\right)+C\left(f_{\overline{\mathcal{P}}}^{d_{i}}\right)-w_{d_{i}}$.

(D) $\bar{d}_{x} \neq d_{y}, d_{x} \neq d_{i}, d_{y}=d_{i}$ : Similarly, we do not need to consider this case.

(E) $d_{x} \neq d_{y}, d_{x} \neq d_{i}, d_{y} \neq d_{i}$ : Similarly, we do not need to consider this case.

Figure 1 shows the illustration of a one-dimensional TCAM minimization problem, where the black bar denotes decision "accept" and the white bar denotes decision "discard". Figure 2 illustrates how the dynamic programming solution works on this example.

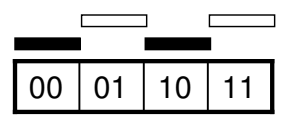

Fig. 1. An example one-dimensional TCAM minimization problem

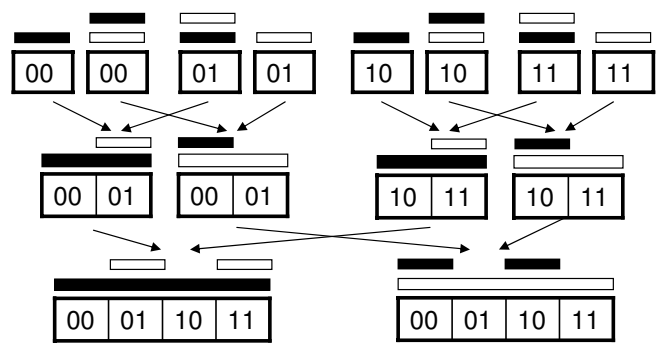

Fig. 2. Illustration of dynamic programming

\section{Multi-Dimensional TCAM Minimization: The Basics}

In this section, we present TCAM Razor, our algorithm for minimizing multi-dimensional prefix packet classifiers. A key idea behind TCAM Razor is processing one dimension at a time using the weighted one-dimensional TCAM minimization algorithm in Section IV to greedily identify a local minimum for the current dimension. Although TCAM Razor is not guaranteed to achieve a global minimum across all dimensions, it does significantly reduce the number of prefix rules in real-life packet classifiers.

\section{A. Conversion to Firewall Decision Diagrams}

To facilitate processing a packet classifier one dimension at a time, we first convert a given packet classifier to an equivalent firewall decision diagram [11].

A Firewall Decision Diagram (FDD) $f$ over fields $F_{1}, \cdots, F_{d}$ is an acyclic and directed graph that has the following five properties:

1) There is exactly one node in $f$ that has no incoming edges. This node is called the root of $f$. The nodes in $f$ that have no outgoing edges are called terminal nodes of $f$.

2) Each node $v$ in $f$ is labeled with a field, denoted $F(v)$, such that

$$
F(v) \in \begin{cases}\left\{F_{1}, \cdots, F_{d}\right\} & \text { if } v \text { is nonterminal } \\ \{\text { accept }, \text { discard }\} & \text { if } v \text { is terminal. }\end{cases}
$$

3) Each edge $e$ in $f$ is labeled with a nonempty set of integers, denoted $I(e)$, such that if $e$ is an outgoing edge of node $v$, then we have $I(e) \subseteq D(F(v))$.

4) A directed path in $f$ from the root to a terminal node is called a decision path. No two nodes on a decision path have the same label.

5) The set of all outgoing edges of a node $v$ in $f$, denoted $E(v)$, satisfies the following two conditions:

a) Consistency: $I(e) \cap I\left(e^{\prime}\right)=\emptyset$ for any two distinct edges $e$ and $e^{\prime}$ in $E(v)$.

b) Completeness: $\bigcup_{e \in E(v)} I(e)=D(F(v))$.

Figure 3 shows an example FDD over two fields $F_{1}, F_{2}$ where the domain of each field is $[0,15]$. Note that in labelling terminal nodes, we use letter " $a$ " as a shorthand for "accept" and letter " $d$ " as a shorthand for "discard".

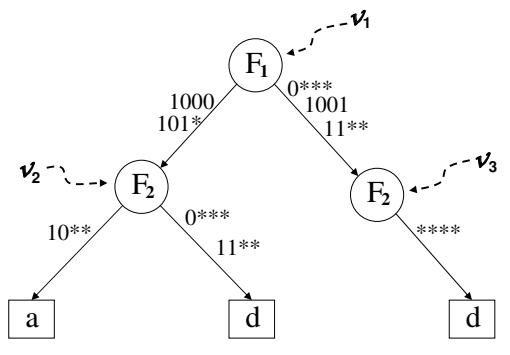

Fig. 3. A packet classifier decision diagram

Given a packet classifier $f_{1}$, we can construct an equivalent FDD $f_{2}$ using the FDD construction algorithm in [17]. An improved FDD construction algorithm is presented in Section VI.

\section{B. Multi-dimensional TCAM Minimization}

We start the discussion of our greedy solution by examining the FDD in Figure 3. We first look at the subgraph rooted at node $v_{2}$. This subgraph can be seen as representing a one-dimension packet classifier over field $F_{2}$. We can use the weighted one-dimensional TCAM minimization algorithm in Section IV to minimize the number of prefix rules for this one-dimensional packet classifier. The algorithm takes the following 3 prefixes as input:

$$
\begin{aligned}
& 10 * * \quad \text { (with decision accept and cost 1), } \\
& 0 * * * \quad \text { (with decision discard and cost 1), }
\end{aligned}
$$


$11 * * \quad$ (with decision discard and cost 1).

The one-dimensional TCAM minimization algorithm will produce a minimum (one-dimensional) packet classifier of two rules as shown in Table III.

\begin{tabular}{|c|c|c|}
\hline Rule \# & $F_{1}$ & Decision \\
\hline 1 & $10^{* *}$ & accept \\
2 & $* * * *$ & discard \\
\hline
\end{tabular}

TABLE III

A MiNimUM PACKET CLASSIFIER CORRESPONDING TO $v_{2}$ IN FIGURE 3

Similarly, from the subgraph rooted at node $v_{3}$, we can get a minimum packet classifier of one rule as shown in Table IV.

\begin{tabular}{|c|c|c|}
\hline Rule \# & $F_{1}$ & Decision \\
\hline 1 & $* * * *$ & discard \\
\hline
\end{tabular}

TABLE IV

A MiNimUM PACKET CLASSIFIER CORRESPONDING TO $v_{3}$ IN FIGURE 3

Next, we look at the root $v_{1}$. As shown in Figure 4, we view the subgraph rooted at $v_{2}$ as a decision with a multiplication factor or cost of 2 , and the subgraph rooted at $v_{3}$ as another decision with a cost of 1 . Thus, the graph rooted at $v_{1}$ can be thought of as a "virtual" one-dimensional packet classifier over field $F_{1}$ where each child has a multiplicative cost.

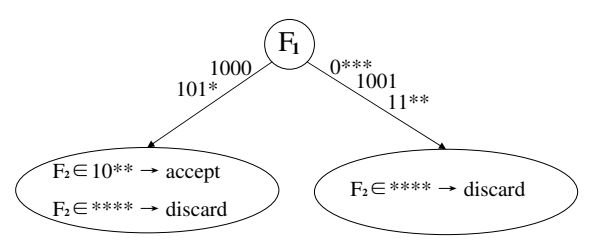

Fig. 4. "Virtual" one-dimensional packet classifier

Now we are ready to use the one-dimensional TCAM minimization algorithm in Section IV to minimize the number of rules for this "virtual" one-dimensional packet classifier. The algorithm takes the following 6 prefixes and associated costs as input:

$\begin{array}{ll}1000 & \left.\text { (with decision } v_{2} \text { and cost } 2\right), \\ 101 * & \left.\text { (with decision } v_{2} \text { and cost } 2\right), \\ 0 * * * & \text { (with decision } v_{3} \text { and cost } 1 \text { ), } \\ 1001 & \text { (with decision } v_{3} \text { and cost } 1 \text { ), } \\ 11 * * & \text { (with decision } v_{3} \text { and cost } 1 \text { ), }\end{array}$

Running the weighted one-dimensional TCAM minimization algorithm on the above input will produce the "virtual" one-dimensional packet classifier of three rules as shown in Table V.

\begin{tabular}{|c|c|c|}
\hline Rule \# & $F_{1}$ & Decision \\
\hline 1 & 1001 & go to node $v_{3}$ \\
2 & $10 * *$ & go to node $v_{2}$ \\
3 & $* * * *$ & go to node $v_{3}$ \\
\hline
\end{tabular}

TABLE V

A MINIMUM PACKET CLASSIFIER CORRESPONDING TO $v_{1}$ IN FIGURE 3

Combining the "virtual" packet classifier in Table V and the two packet classifiers in Table III and IV, we get a packet classifier of 4 rules as shown in Table VI.

\begin{tabular}{|c|cc|c|}
\hline Rule \# & $F_{1}$ & $F_{2}$ & Decision \\
\hline 1 & 1001 & $* * * *$ & discard \\
2 & $10 * *$ & $10 * *$ & accept \\
3 & $10 * *$ & $* * * *$ & discard \\
4 & $* * * *$ & $* * * *$ & discard \\
\hline
\end{tabular}

TABLE VI

PACKET CLASSIFIER GENERATED FROM THE FDD IN FIGURE 3

\section{Removing Redundant Rules}

Next, we observe that rule $r_{3}$ in the packet classifier in Table VI is redundant. If we remove rule $r_{3}$, all the packets that used to be resolved by $r_{3}$ (that is, all the packets that match $r_{3}$ but do not match $r_{1}$ and $r_{2}$ ) are now resolved by rule $r_{4}$, and $r_{4}$ has the same decision as $r_{3}$. Therefore, removing rule $r_{3}$ does not change the semantics of the packet classifier. Redundant rules in a packet classifier can be removed using the algorithms in [18]. Finally, after removing redundant rules, we get a packet classifier of 3 rules from the FDD in Figure 3.

\section{The Algorithm}

To summarize, TCAM Razor, our multi-dimensional TCAM minimization algorithm, consists of the following four steps:

1) Convert the given packet classifier to an equivalent FDD.

2) Use the FDD reduction algorithm described in the next section to reduce the size of the FDD. This step will be explained in more detail in the next section.

3) Generate a packet classifier from the FDD in the following bottom up fashion. For every terminal node, assign a cost of 1 . For a non-terminal node $v$ with $z$ outgoing edges $\left\{e_{1}, \cdots, e_{z}\right\}$, formulate a one-dimensional TCAM minimization problem as follows. For every prefix $\mathcal{P}$ in the label of edge $e_{j},(1 \leq j \leq z)$, we set the decision of $\mathcal{P}$ to be $j$, and the cost of $\mathcal{P}$ to be the cost of the node that edge $e_{j}$ points to. For node $v$, we use the weighted one-dimensional TCAM minimization algorithm in Section IV to compute a one-dimensional prefix packet classifier with the minimum cost. We then assign this minimum cost to the cost of node $v$. After the root node is processed, generate a packet classifier using the prefixes computed at each node in a depth first traversal of the FDD. The cost of the root indicates the total number of prefix rules in the resulting packet classifier.

4) Remove all the redundant rules from the resulting packet classifier.

\section{Vi. Multi-dimensional TCAM Minimization: The OPTIMIZATION TECHNIQUES}

In this section, we discuss the following two optimization techniques that we implemented to reduce the running time and memory usage of TCAM Razor: lazy copying in FDD construction and hashing in FDD reduction.

\section{A. Lazy Copying in FDD Construction}

The FDD construction algorithm in [17] performs deep copying of subgraphs when splitting edges. This is inefficient in terms of both running time and memory usage. In TCAM Razor, we use the technique of lazy copying, which is explained as follows. Consider the subgraph (of an FDD) in Figure 5. The root of this subgraph is $v$, and $v$ has $k$ outgoing edges $e_{1}, e_{2}, \cdots, e_{k}$, which point to the subgraphs $g_{1}, g_{2}, \cdots, g_{k}$ respectively. When we need to make 
another copy of this subgraph, instead of making a deep copy of the whole subgraph, we only make another copy of the root of the subgraph. Let $v^{\prime}$ denote the new node. Node $v^{\prime}$ has the same label as $v$, and also has $k$ outgoing edges $e_{1}^{\prime}, e_{2}^{\prime}, \cdots, e_{k}^{\prime}$, where each $e_{i}^{\prime}$ has the same label $I\left(e_{i}\right)$ as $e_{i}$, and also points to the same subgraph $g_{i}$ that $e_{i}$ points to.

Each time we need to modify a node $v$, we first need to check its in-degree (i.e., the number of edges that point to $v$ ): if its indegree is 1 , then we can directly modify $v$; if its indegree is greater than 1 , then we need to first make a lazy copy of the subgraph rooted at $v$, and then modify the new node $v^{\prime}$. To the outside, lazy copying looks like deep copying, but it reduces unnecessary copying of subgraphs (and promotes the sharing of common subgraphs) in the constructed FDD as much as possible.

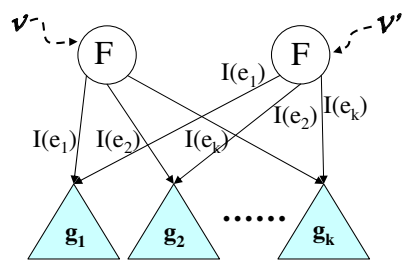

Fig. 5. Lazing copying of subgraphs

Figure 6 shows the process of appending rule $\left(F_{1} \in 0000\right) \wedge$ $\left(F_{2} \in 010 *\right) \wedge\left(F_{3} \in 0 * * *\right) \rightarrow d$ to node $v_{1}$ of the partial FDD in (a). A partial FDD is a diagram that has all the properties of an FDD except the completeness property.

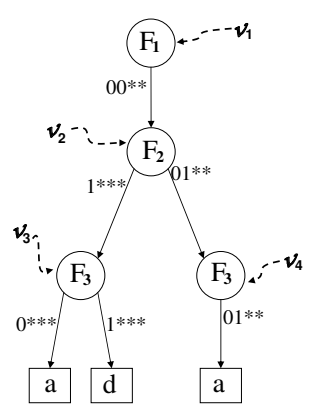

(a) A partial FDD

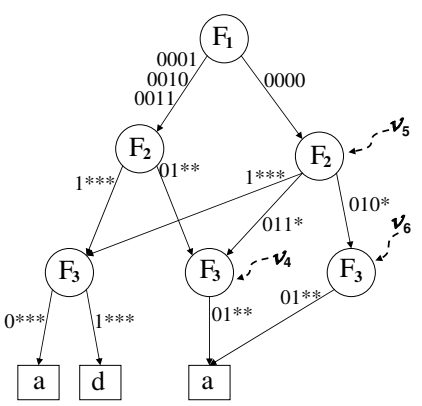

(c) After appending $\left(F_{2} \in 010 *\right) \wedge$ $\left(F_{1} \in 0 * * *\right) \rightarrow d$ to $v_{5}$

Fig. 6. Example of lazing copying in the improved FDD construction algorithm

\section{B. Hashing in FDD Reduction}

To further reduce the number of rules generated by our algorithm, after we convert a packet classifier to an equivalent FDD, we need to reduce the size of the FDD. An FDD $f$ is reduced if and only if it satisfies the following three conditions: (1) no two nodes in $f$ are isomorphic; (2) no two nodes have more than one edge between them; (3) no node in $f$ has only one outgoing edge. Two nodes $v$ and $v^{\prime}$ in an FDD are isomorphic if and only if $v$ and $v^{\prime}$ satisfy one of the following two conditions: (1) both $v$ and $v^{\prime}$ are terminal nodes with identical labels; (2) both $v$ and $v^{\prime}$ are nonterminal nodes and there is a one-to-one correspondence between the outgoing edges of $v$ and the outgoing edges of $v^{\prime}$ such that every pair of corresponding edges have identical labels and they both point to the same node.

We next show a motivating example in which FDD reduction helps to reduce the number of prefix rules generated from an FDD. Consider the two equivalent FDDs in Figure 7 where (a) is nonreduced and (b) is reduced. If we run our multi-dimensional TCAM minimization algorithm on the two FDDs in Figure 7(a) and 7(b), we will produce 4 prefix rules as shown in Table VII and 2 prefix rules as shown in Table VIII, respectively.

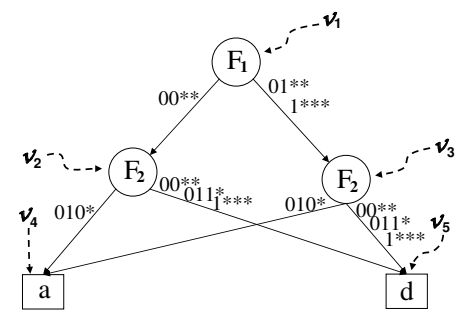

(a) Before FDD reduction

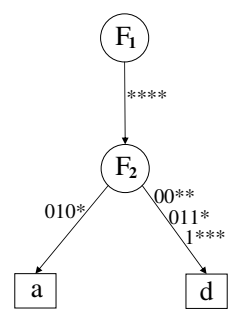

(b) After FDD reduction
Fig. 7. Example of FDD reduction

\begin{tabular}{|c|cc|c|}
\hline Rule $\#$ & $F_{1}$ & $F_{2}$ & Decision \\
\hline 1 & $00^{* *}$ & $010^{*}$ & accept \\
2 & $00 * *$ & $* * * *$ & discard \\
3 & $* * * *$ & $010^{*}$ & accept \\
4 & $* * * *$ & $* * * *$ & discard \\
\hline
\end{tabular}

TABLE VII

RULES GENERATED FROM THE FDD IN FIGURE 7(A)

\begin{tabular}{|c|cc|c|}
\hline Rule \# & $F_{1}$ & $F_{2}$ & Decision \\
\hline 1 & $* * * *$ & $010^{*}$ & accept \\
2 & $* * * *$ & $* * * *$ & discard \\
\hline
\end{tabular}

TABLE VIII

RULES GENERATED FROM THE FDD IN FIGURE 7(B)

A brute force deep comparison algorithm for FDD reduction was proposed in [11]. In TCAM Razor, we use a more efficient FDD reduction algorithm that processes the nodes level by level from the terminal nodes to the root node using signatures to speed up comparisons.

1) Starting from the bottom level, at each level, we compute a signature for each node at that level. For a terminal node $v$, set $v$ 's signature to be its label. For a non-terminal node $v$, suppose $v$ has $k$ children $v_{1}, v_{2}, \cdots, v_{k}$, in increasing order of signature $\left(\operatorname{Sig}\left(v_{i}\right)<\operatorname{Sig}\left(v_{i+1}\right)\right.$ for $\left.1 \leq i \leq k-1\right)$, and the edge between $v$ and its child $v_{i}$ is labeled with $E_{i}$, a sequence of non-overlapping prefixes in increasing order. Set 
the signature of node $v$ as follows:

$$
\operatorname{Sig}(v)=h\left(\operatorname{Sig}\left(v_{1}\right), E_{1}, \cdots, \operatorname{Sig}\left(v_{k}\right), E_{k}\right)
$$

where $h$ is a hash function.

2) After we have assigned signatures to all nodes at a given level, we search for isomorphic subgraphs as follows. For every pair of nodes $v_{i}$ and $v_{j}(1 \leq i \neq j \leq k)$ at this level, if $\operatorname{Sig}\left(v_{i}\right) \neq \operatorname{Sig}\left(v_{j}\right)$, then we can conclude that $v_{i}$ and $v_{j}$ are not isomorphic; otherwise, we explicitly determine if $v_{i}$ and $v_{j}$ are isomorphic. If $v_{i}$ and $v_{j}$ are isomorphic, we delete node $v_{j}$ and its outgoing edges, and redirect all the edges that point to $v_{j}$ to point to $v_{i}$. Further, we eliminate double edges between node $v_{i}$ and its parents.

For example, the signatures of the non-root nodes in Figure 7(a) are computed as follows:

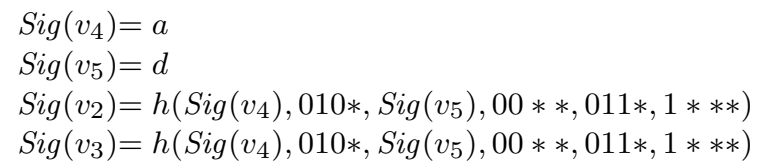

\section{EXPERIMENTAL RESULTS}

In this section, we evaluate the effectiveness and efficiency of TCAM Razor on both real-life and synthetic packet classifiers. Note that in cases where TCAM Razor cannot produce smaller packet classifiers than redundancy removal alone, TCAM Razor will return the classifier produced by redundancy removal. Thus, TCAM Razor always performs at least as well as redundancy removal.

\section{A. Methodology}

We first define the metrics that we used to measure the effectiveness of TCAM Razor and the redundancy removal technique by Liu and Gouda [18]. In this paragraph, $f$ denotes a packet classifier, $S$ denotes a set of packet classifiers, and $A$ denotes either TCAM Razor or the redundancy removal technique. We then let $|f|$ denote the number of rules in $f, A(f)$ denote the prefix classifier produced by applying $A$ on $f$, and $\operatorname{Direct}(f)$ denote the prefix classifier produced by applying direct prefix expansion on $f$. We define the following four metrics for assessing the performance of $A$ on a set of classifiers $S$.

- The average compression ratio of $A$ over $S=\frac{\Sigma_{f \in S} \frac{|A(f)|}{|\operatorname{Direct}(f)|}}{|S|}$.

- The total compression ratio of $A$ over $S=\frac{\Sigma_{f \in S}|A(f)|}{\Sigma_{f \in S}|\operatorname{Direct}(f)|}$.

- The average expansion ratio of $A$ over $S=\frac{\Sigma_{f \in S} \frac{|A(f)|}{|f|}}{|S|}$.

- The total expansion ratio of $A$ over $S=\frac{\Sigma_{f \in S}|A(f)|}{\Sigma_{f \in S}|f|}$.

We next define notation to specify TCAM Razor and its dependence on the variable order used to convert a packet classifier into an equivalent FDD. There are $5 !=120$ different permutations of the five packet fields (source IP address, destination IP address, source port number, destination port number, and protocol type). We number these permutations from 1 to 120 , and we use the notation TCAM Razor $(i)$ to denote TCAM Razor using permutation $i$, and for a given packet classifier $f$, we use TCAM Razor(B) to denote TCAM Razor using the best of the 120 permutations for $f$.

\section{B. Effectiveness on Real-life Packet Classifiers}

We first defined a set $R L$ of 17 real-life packet classifiers that we performed experiments on. We actually obtained 42 real-life packet classifiers from distinct network service providers that range

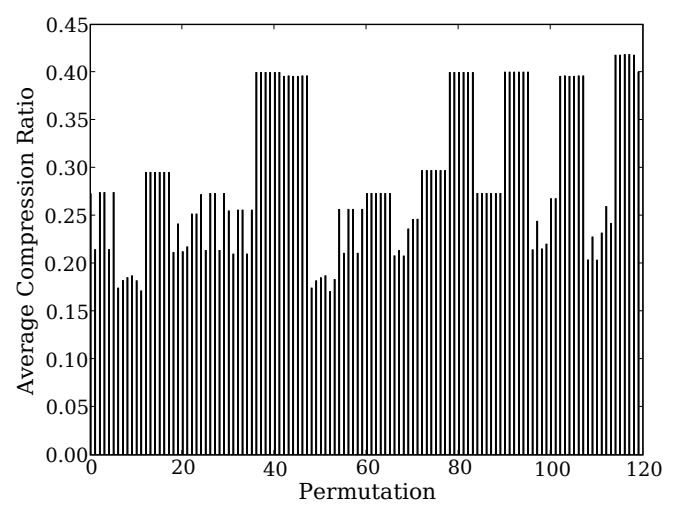

Fig. 8. The average compression ratio for each permutation

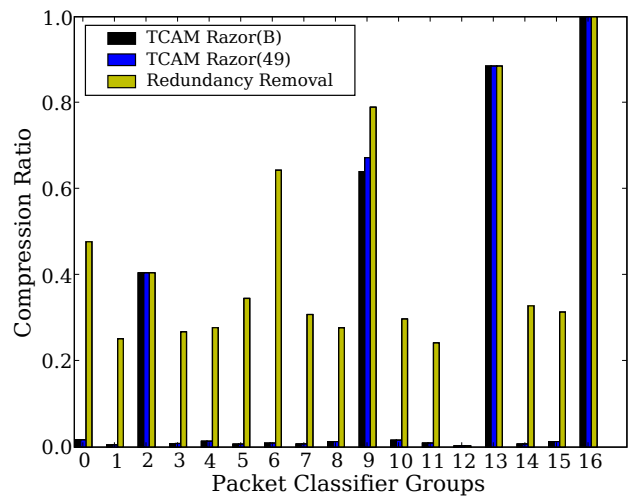

Fig. 9. Compression ratios of real-life packet classifier groups

in size from dozens to hundreds of rules. Although this collection of classifiers was diverse, some classifiers from the same network service provider had similar structure and exhibited similar results under TCAM Razor. To prevent this repetition from skewing the performance data, we divided the 42 packet classifiers into 17 structurally distinct groups, and we chose one representative from each of the 17 groups to form the set $R L$.

1) Variable Ordering: A question that naturally arises is: which variable order achieves the best average compression ratio? To answer this question, for each permutation $i$, we computed the average compression ratio that TCAM $\operatorname{Razor}(i)$ achieved over $R L$. The results are shown in Figure 8. The maximum average compression ratio is $41.8 \%$. Furthermore, more than half of the permutations have average compression ratios below $29.1 \%$, and four permutations have average compression ratios below $18.3 \%$. Of these four permutations, permutation 49 (source IP address, protocol type, destination IP address, destination port number, source port number) is the best with an average compression ratio of $18.2 \%$.

The next natural question to ask is: is permutation 49 the best order for most packet classifiers? The answer for $R L$ is yes. In Figure 9, for each packet classifier in $R L$, we show the compression ratios of TCAM Razor(B), TCAM Razor(49), and redundancy removal. The results show that permutation 49 achieves almost the best compression ratio for each packet classifier group.

2) Compression Ratio: Our experimental results clearly demonstrate that TCAM Razor outperforms just redundancy removal [18]. For example, the average compression ratios of TCAM Razor(49) and redundancy removal over $R L$ are $18.2 \%$ and $41.8 \%$ respec- 


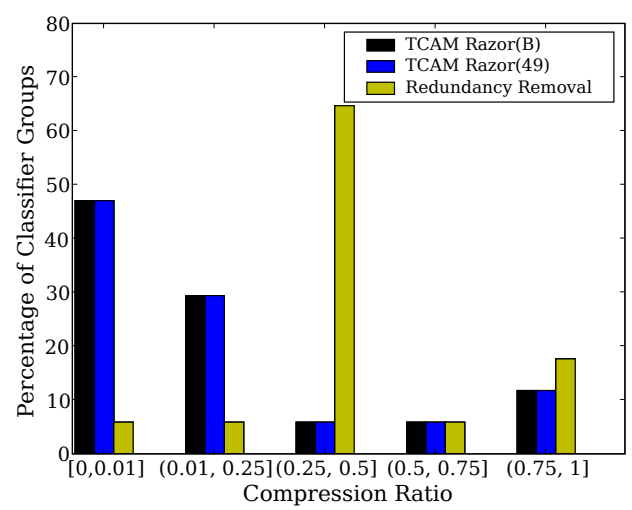

Fig. 10. Distribution of real-life packet classifiers by compression ratio

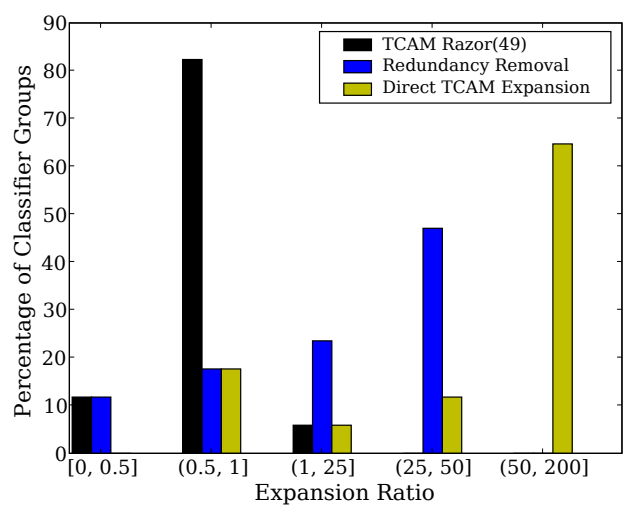

Fig. 11. Distribution of real-life packet classifiers by expansion ratio

tively. Similarly, the total compression ratios of TCAM Razor(49) and redundancy removal over $R L$ are $3.9 \%$ and $35 \%$ respectively. Figure 9 shows that TCAM Razor(49) significantly outperforms redundancy removal on 13 of the 17 real-life packet classifier groups. TCAM Razor(49) has a compression ratio of less than or equal to $1 \%$ on 8 of the 17 classifier groups in $R L$. Figure 10 shows the distribution of compression ratios achieved by TCAM Razor and redundancy removal alone on $R L$.

3) Expansion Ratio: We observe similar results for expansion ratio. The average expansion ratios for TCAM Razor(49), redundancy removal, and direction prefix expansion over $R L$ are 0.754 , 19.877 , and 69.870 , respectively. The total expansion ratio for TCAM Razor(49), redundancy removal, and direct prefix expansion over $R L$ are $0.797,7.147$, and 20.414 , respectively.

Figure 11 shows the distribution of expansion ratios for the following three algorithms: TCAM Razor(49), redundancy removal, and direct prefix expansion. Prefix expansion is a real issue as over $60 \%$ of our packet classifiers have an expansion ratio of over 50 if we use direct prefix expansion. The experimental data also suggests that TCAM Razor addresses the prefix expansion issue well as TCAM Razor(49) has an expansion ratio of at most 1 on 16 of the 17 real-life packet classifier groups in our experiments, and TCAM Razor(49) has an expansion ratio of 1.07 on the 17th real-life packet classifier.

\section{Comparison with Dong et al. [8]}

It is difficult to compare our results directly with those of Dong et al. [8] because we do not have access to their programs or

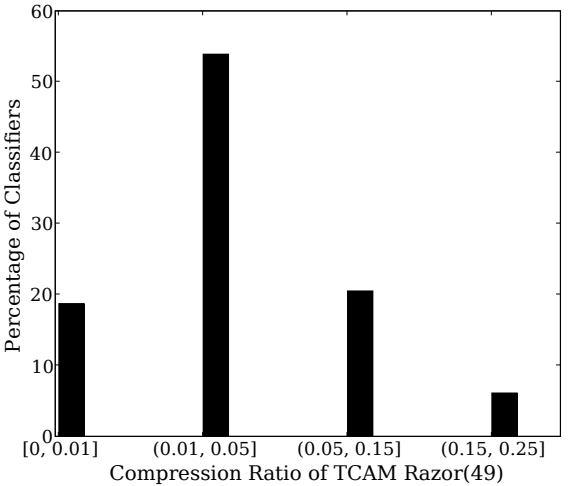

Fig. 12. Distribution of synthetic packet classifiers by compression ratio

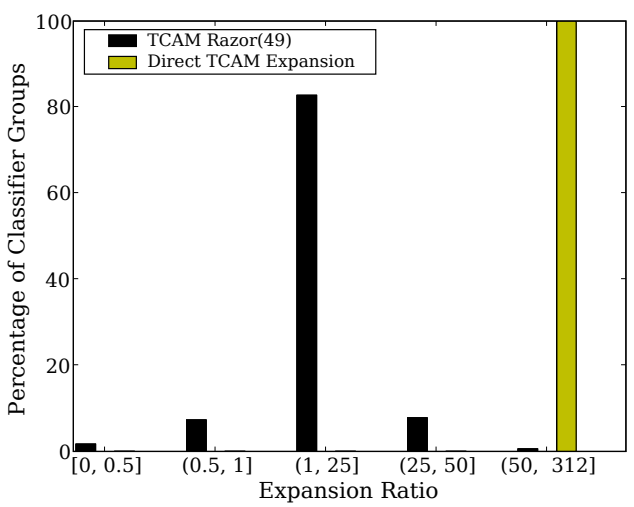

Fig. 13. Distribution of synthetic packet classifiers by expansion ratio

the packet classifiers they experimented with. However, TCAM Razor(49) has a total compression ratio of $3.9 \%$ on our reallife packet classifiers. In contrast, Dong et al. reported a total compression ratio ${ }^{1}$ of $54 \%$ on their real-life packet classifiers.

\section{Effectiveness on Synthetic Packet Classifiers}

Packet classifier rules are considered confidential due to security concerns. Thus, it is difficult to get many real-life packet classifiers to experiment with. To address this issue and further evaluate the performance of TCAM Razor, we generated $S Y N$, a set of synthetic packet classifiers of 18 sizes, where each size has 100 independently generated classifiers.

Every predicate of a rule in our synthetic packet classifiers has five fields: source IP address, destination IP address, source port number, destination port number, and protocol type. We first randomly generated a list of values for each field. For IP addresses, we generated a random class $\mathrm{C}$ address; for ports we generated a random interval; for protocols, we generated a random protocol number. Given these lists, we generated a list of predicates by taking the cross product of all these lists. We added a final default predicate to our list. Finally, we randomly assigned one of two decisions, accept or deny, to each predicate to make a complete packet classifier.

Distributions of compression ratios and expansion ratios over $S Y N$ are shown in Figures 12 and 13. The average compression

${ }^{1}$ By clarifying with the authors of [8], the term "average compression ratio" used but not defined in [8] is actually what we define as "total compression ratio" in this paper. 
ratio of TCAM Razor(49) over $S Y N$ is $4.6 \%$, the average expansion ratio of TCAM Razor(49) over $S Y N$ is 8.737, the total compression ratio of TCAM Razor(49) over $S Y N$ is $1.6 \%$, and the total expansion ratio of TCAM Razor(49) over $S Y N$ is 3.082 .

\section{E. Efficiency of TCAM Razor}

We implemented TCAM Razor using Visual Basic on Microsoft .Net framework 2.0. In our experiments, we first ran TCAM Razor on real-life packet classifiers, and then we stress tested TCAM Razor on a large number of big synthetic packet classifiers. Our experiments were carried out on a desktop PC running Windows $\mathrm{XP}$ with $1 \mathrm{G}$ memory and a single $2.2 \mathrm{GHz}$ AMD Opteron 148 processor. Table IX shows the total running time of TCAM Razor(49) for three representative packet classifiers. Figure 14 displays the average total running time of TCAM Razor(49) on our synthetic packet classifiers as a function of the number of original rules along with the standard deviation.

\begin{tabular}{|c|c|}
\hline Number of Original Rules & TCAM Razor Running Time (seconds) \\
\hline 42 & 0.2 \\
\hline 87 & 0.9 \\
\hline 661 & 31.9 \\
\hline
\end{tabular}

TABLE IX

SAMPLE RUNNING TIME DATA FOR REAL-LIFE PACKET CLASSIFIERS

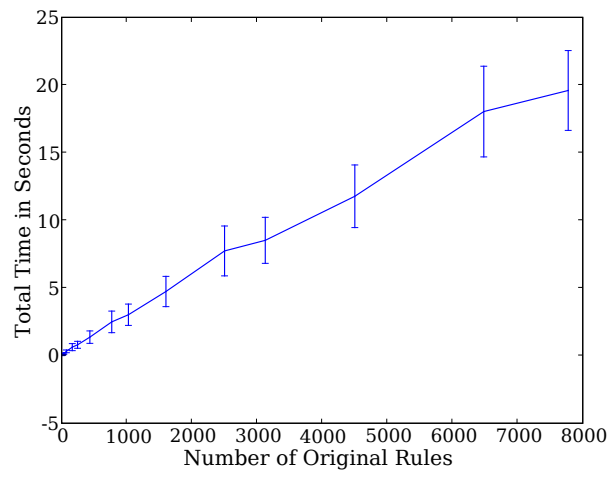

Fig. 14. Total runtime vs. number of original rules

\section{CONCLUSIONS}

TCAMs have become the de facto industry standard for packet classification. However, as the rules in packet classifiers grow in number and complexity, the viability of TCAM-based solutions is threatened by the problem of prefix expansion. In this paper, we propose TCAM Razor, a systematic approach to minimizing TCAM rules for packet classifiers. While TCAM Razor does not always produce optimal packet classifiers, in our experiments with 17 structurally distinct real-life packet classifier groups, TCAM Razor reduced the number of TCAM entries needed by an average of $81.8 \%$ percent and a total of $96.1 \%$. In fact, TCAM Razor experienced no expansion for 16 of the 17 real-life packet classifier groups. While it is difficult to perform a direct comparison with Deng et al.'s approach [8], it appears that TCAM Razor performs significantly better with a total compression ratio of $3.9 \%$ as compared with a total compression ratio of $54 \%$. Finally, unlike other solutions that require modifying TCAM circuits or packet processing hardware, TCAM Razor can be deployed today by network administrators and ISPs to cope with prefix expansion.

\section{REFERENCES}

[1] Cypress semiconductor corp. content addressable memory. http://www.cypress.com/.

[2] Integrated device technology, inc. content addressable memory. http://www.idt.com/.

[3] Netlogic microsystems. content addressable memory. http://www.netlogicmicro.com/.

[4] A guide to search engines and networking memory. http://www.linleygroup.com/pdf/NMv4.pdf, November 2006.

[5] D. A. Applegate, G. Calinescu, D. S. Johnson, H. Karloff, K. Ligett, and J. Wang. Compressing rectilinear pictures and minimizing access control lists. In Proceedings of the Proceedings of ACM-SIAM Symposium on Discrete Algorithms (SODA), January 2007.

[6] F. Baboescu, S. Singh, and G. Varghese. Packet classification for core routers: Is there an alternative to CAMs? In Proceedings of IEEE INFOCOM, 2003.

[7] F. Baboescu and G. Varghese. Scalable packet classification. In Proceedings of ACM SIGCOMM, pages 199-210, 2001.

[8] Q. Dong, S. Banerjee, J. Wang, D. Agrawal, and A. Shukla. Packet classifiers in ternary CAMs can be smaller. In Proceedings of SIGMETRICS, pages 311-322, 2006.

[9] R. Draves, C. King, S. Venkatachary, and B. Zill. Constructing optimal IP routing tables. In Proceedings of IEEE INFOCOM, pages 88-97, 1999.

[10] A. Feldmann and S. Muthukrishnan. Tradeoffs for packet classification. In Proceedings of 19th IEEE INFOCOM, Mar. 2000.

[11] M. G. Gouda and A. X. Liu. Structured firewall design. Computer Networks Journal, 51(4):1106-1120, March 2007.

[12] P. Gupta and N. McKeown. Packet classification on multiple fields. In Proceedings of ACM SIGCOMM, pages 147-160, 1999.

[13] P. Gupta and N. McKeown. Packet classification using hierarchical intelligent cuttings. In Proceedings of Hot Interconnects VII, Aug. 1999.

[14] P. Gupta and N. McKeown. Algorithms for packet classification. IEEE Network, 15(2):24-32, 2001.

[15] T. V. Lakshman and D. Stiliadis. High-speed policy-based packet forwarding using efficient multi-dimensional range matching. In Proceedings of ACM SIGCOMM, pages 203-214, 1998.

[16] K. Lakshminarayanan, A. Rangarajan, and S. Venkatachary. Algorithms for advanced packet classification with ternary cams. In Proceedings of the ACM SIGCOMM, pages 193 - 204, August 2005.

[17] A. X. Liu and M. G. Gouda. Diverse firewall design. In Proceedings of the International Conference on Dependable Systems and Networks (DSN-04), pages 595-604, June 2004.

[18] A. X. Liu and M. G. Gouda. Complete redundancy detection in firewalls. In Proceedings of 19th Annual IFIP Conference on Data and Applications Security, LNCS 3654, S. Jajodia and D. Wijesekera Ed., Springer-Verlag, pages 196-209, August 2005.

[19] H. Liu. Efficient mapping of range classifier into ternary-cam. In Proceedings of the Hot Interconnects, pages 95- 100, 2002.

[20] M. H. Overmars and A. F. van der Stappen. Range searching and point location among fat objects. Journal of Algorithms, 21(3):629-656.

[21] L. Qiu, G. Varghese, and S. Suri. Fast firewall implementations for software-based and hardware-based routers. In Proceedings the 9th International Conference on Network Protocols (ICNP), 2001.

[22] S. Singh, F. Baboescu, G. Varghese, and J. Wang. Packet classification using multidimensional cutting. In Proceedings of ACM SIGCOMM, 2003.

[23] E. Spitznagel, D. Taylor, and J. Turner. Packet classification using extended tcams. In Proceedings of the 11th IEEE International Conference on Network Protocols (ICNP), pages 120- 131.

[24] V. Srinivasan, G. Varghese, S. Suri, and M. Waldvogel. Fast and scalable layer four switching. In Proceedings of ACM SIGCOMM, pages 191-202, 1998.

[25] S. Suri, T. Sandholm, and P. Warkhede. Compressing two-dimensional routing tables. Algorithmica, 35:287-300, 2003.

[26] D. E. Taylor. Survey \& taxonomy of packet classification techniques. ACM Computing Surveys, 37(3):238-275, 2005.

[27] J. van Lunteren and T. Engbersen. Fast and scalable packet classification. IEEE Journals on Selected Areas in Communications, 21(4):560- 571, 2003.

[28] M. Waldvogel, G. Varghese, J. Turner, and B. Plattner. Scalable high speed IP routing lookups. In Proceedings of ACM SIGCOMM, pages 25-36, September 1997.

[29] T. Y. C. Woo. A modular approach to packet classification: Algorithms and results. In Proceedings of IEEE INFOCOM, pages 1213-1222, 2000.

[30] F. Yu, T. V. Lakshman, M. A. Motoyama, and R. H. Katz. Ssa: A power and memory efficient scheme to multi-match packet classification. In Proceedings of the Symposium on Architectures for Networking and Communications Systems (ANCS), pages 105-113, October 2005. 